\title{
Caracterização da sífilis em gestantes no município de Codó - Maranhão no período de 2012 a 2017
}

\author{
Characterization of syphilis in pregnant in a Codó - Maranhão municipality in the period \\ from 2012 to 2017
}

Caracterización de la sífilis en gestantes en un municipio de Codó - Maranhão en el período de 2012 a 2017

Karine Teodora Alves de Oliveira ${ }^{1}$, Lucas Eduardo Morais ${ }^{1}$, Joseana Martins Soares de Rodrigues Leitão ${ }^{1}$, Roseane Mara Cardoso Lima Verde ${ }^{2}$, Matheus Hipólito do Nascimento ${ }^{3}$, Thiago Rodrigues Oliveira ${ }^{4}$, Pedro Simão da Silva Azevedo ${ }^{4}$, Arionildo Batista Moraes ${ }^{4}$, Evaldo Hipólito de Oliveira ${ }^{1,5 *}$.

\section{RESUMO}

Objetivo: analisar e descrever a distribuição dos casos notificados de sífilis em gestante no período de 2012 a 2017, no município de Codó. Metodologia: um estudo retrospectivo analítico, com base em levantamento de casos. O estudo compreende todos os casos notificados de sífilis em gestantes entre 2012 a 2017 no Município de Codó - Maranhão. Os dados foram coletados no Sistema de Informação de Agravos de Notificação - SINAN, contidos no Núcleo de Vigilância Epidemiológica (NVE) do município de Codó Maranhão. Resultados: no período em estudo, foram registrados 253 casos confirmados de sífilis em gestantes no município de Codó-MA, caracterizando uma média anual de 42,16 casos. O maior número de casos confirmados de sífilis em gestante foi no ano de 2016 ( $n=68 ; 26,88 \%)$ e 2015 ( $n=56 ; 22,14 \%)$; a faixa etária com predominância dos casos confirmados de sífilis em gestante foram da faixa etária entre 20 e 39 anos ( $n=169 ; 66,80 \%)$; a sífilis primária em gestantes foi a mais notificada ( $n=144 ; 56,92 \%)$. A segunda mais notificada foi a sífilis secundária $(n=26 ; 10,28 \%) ; 197(77,86 \%)$ foram considerados reativos segundo teste treponêmico e, apenas $4(1,58 \%)$ foram considerados como não reativo; o total de casos confirmados como reativo pelo teste não treponêmico foi $168(66,40 \%)$, enquanto os casos não reativos corresponderam a um percentual de 1,98\% $(n=5)$. Conclusão: Os registros de notificações de sífilis em gestantes são instrumentos valiosos para monitoramento e avaliação da assistência prestada às gestantes com diagnóstico de sífilis. Por isso, é importante que a gestão e a assistência entendam a necessidade do preenchimento adequado das informações do formulário e da alimentação do SINAM. Também é necessária a atualização dos profissionais para a assistência às gestantes com sífilis e a importância da notificação. $O$ grande desafio para a saúde pública é aumentar a cobertura e a qualidade do pré-natal, incluindo o parceiro sexual ativamente nas consultas, ampliar o diagnóstico laboratorial da sífilis e o consequente tratamento durante o pré-natal e no momento do parto.

Palavras - Chaves: Sífilis, Gestantes, Saúde Pública.

\section{ABSTRACT}

Aim: to analyze and describe the management of reported cases of syphilis in pregnant women from 2012 to 2017, in Codó. Methodology: a retrospective analytical study, based on new cases. The study includes all reported cases of syphilis in pregnant women between 2012 and 2017 in of Codó - Maranhão. The data were collected in the Information System of Notification Diseases - SINAN, contained in the Nucleus of

\footnotetext{
${ }^{1}$ Centro Universitário Santo Agostinho, Teresina, PI.

2 Programa de Pós-Graduação em Engenharia Biomédica - PPGEB- Universidade Brasil, São Paulo, SP.

${ }^{3}$ Centro Universitário UNIEURO, Brasília, DF

${ }^{4}$ Biomédico pelo Centro Universitário UNINOVAFAPI, Teresina, PI.

5 Universidade Federal do Piauí, Teresina, PI. *E-mail: evaldohipolito@gmail.com
} 
Epidemiological Surveillance (NVE) of Codó - Maranhão. Results: 253 confirmed cases of syphilis were included in pregnant women in the municipality of Codó-MA, with an annual average of 42.16 cases. The highest number of confirmed cases of syphilis in pregnant women was $2016(n=68,26.88 \%)$ and $2015(n=$ $56,22.14 \%$ ); The age group predominated with confirmed cases of syphilis in pregnant women in the age group between 20 and 39 years $(n=169,66.80 \%)$; primary syphilis in pregnant women was more frequently reported $(n=144 ; 56.92 \%)$. The second most reported was secondary syphilis $(n=26 ; 10.28 \%) ; 197(77.86 \%)$ were consecutive according to the treponemal test, and only $4(1.58 \%)$ were considered non-reactive; 168 $(66.40 \%)$, while non-reactive cases were $1.98 \%(n=5)$. Conclusion: Records of syphilis reports are valuable tools for the monitoring and evaluation of care provided to pregnant women diagnosed with syphilis. Therefore, it is important that the management of a school be done to fill out the information about the form and the feeding of SINAM. An update of the professionals is also required for an assessment of syphilis management and the importance of notification. The major challenge for public health is to increase the coverage and quality of prenatal care, including actively delivering sexual intercourse at the clinic, expanding the laboratory diagnosis of syphilis, and the consequent treatment during prenatal care and no delivery.

Key Words: Syphilis, Pregnant women, Public health.

\section{RESUMEN}

Objetivo: analizar y describir la gestión de casos notificados de sífilis en gestante en el período de 2012 a 2017, en el municipio de Codó. Metodología: un estudio retrospectivo analítico, basado en nuevos casos. El estudio comprende todos los casos notificados de sífilis en gestantes entre 2012 y 2017 en el Municipio de Codó - Maranhão. Los datos fueron recolectados en el Sistema de Información de Agravios de Notificación SINAN, contenidos en el Núcleo de Vigilancia Epidemiológica (NVE) del municipio de Codó - Maranhão. Resultados: en el período en estudio, se incluyeron 253 casos confirmados de sífilis en gestantes en el municipio de Codó-MA, con una media anual de 42,16 casos. El mayor número de casos confirmados de sífilis en gestante fue en el año $2016(n=68,26,88 \%)$ y $2015(n=56 ; 22,14 \%)$; La edad media de los casos confirmados de sífilis en gestante en el grupo de edad entre 20 y 39 años $(n=169 ; 66,80 \%)$; se notificó una sífilis primaria en gestantes $(n=144,56,92 \%)$. La segunda más notificada fue la sífilis secundaria $(n=26$; 10,28\%); 197 (77,86\%) fueron consecutivos según test treponémico y, apenas $4(1,58 \%)$ fueron considerados como no reactivos; el total de casos confirmados como prueba no treponémico fue $168(66,40 \%)$, mientras que los casos no reactivos respondieron a un porcentaje del 1,98\% $(n=5)$. Conclusión: Los registros de notificaciones de sífilis son instrumentos valiosos para el monitoreo y evaluación de la asistencia prestada a las gestantes con diagnóstico de sífilis. Por eso, es importante que la gestión de una escuela sea hecha para el llenado de las informaciones sobre el formulario y la alimentación del SINAM. También es necesaria una actualización de los profesionales para una evaluación de gestiones con sífilis y la importancia de la notificación. El gran desafío para la salud pública es aumentar la cobertura y la calidad del prenatal, incluyendo el parto sexual activamente en las consultas, ampliar el diagnóstico de laboratorio de la sífilis y el consiguiente tratamiento durante el prenatal y ningún momento del parto.

Palabras - claves: Sífilis, Las mujeres embarazadas, Salud pública.

\section{INTRODUÇÃO}

Considera uma doença de infecção contagiosa sistêmica, a sífilis tem como causa bactéria Treponema pallidum, cuja principal via de transmissão é a sexual e por outros contatos íntimos, com maior frequência em mulheres de idade fértil, podendo ocorrer na gravidez e ser passada para o filho, transmissão vertical, sendo nesse caso conhecida como sífilis congênita (SUTO et al., 2016). Logo, trata-se de um problema de saúde pública tanto, porém é fácil de ser tratada, haja vista a gestante portadora passe por diagnóstico no pré-natal e seja submetida ao tratamento adequado, juntamente com seu parceiro, caso isso não ocorra, pode ter aborto, partos prematuros e sequelas (COSTA et al., 2013; SUTO et al., 2016; FURTADO et al., 2018).

Quanto mais cedo for iniciado o tratamento melhores resultados serão alcançados, assim os objetivos iniciais são fortalecer o planejamento e a avaliação de medidas de prevenção e controle, particularmente a transmissão vertical do agente etiológico. Para controlar de forma efetiva a doença necessita-se de início a triagem sorológica, uma vez que a qualidade da assistência pré-natal e ao parto são importantes 
determinantes na redução da transmissão materno-infantil, este é um dos principais entraves para a alta incidência de sífilis em gestantes e sífilis congênita ainda é um desafio para os serviços de saúde (GRUMACH et al., 2007; CAVALCANTE; PEREIRA; CASTRO, 2017).

Dessa forma, chama-se a atenção para o quão é importante o pré-natal, uma vez que as principais estratégias para diminuir o número de casos da doença estão voltadas a garantir que todas as gestantes recebam assistência pré-natal, e a triagem de sífilis seja incluída como rotina a todas as mulheres e aos parceiros. A doença pode apresentar ou não sintomas, e quando diagnostica deve ser notificada à vigilância epidemiológica e tratados pelo sistema de saúde (REZENDE; BARBOSA, 2015).

Como observado por Vasconcelos et al (2017), ainda persiste o insucesso na prevenção e diagnostico cedo da enfermidade, isso se dá, principalmente, em função de: as barreiras para o acesso pleno aos serviços de saúde, a falta de solicitação do exame sorológico para gestante durante o pré-natal e a não abordagem para o tratamento e avaliação dos parceiros sexuais das mulheres com resultado positivo, isso acontece com maior proeminência em cidades do interior do Nordeste. Baseado no exposto, o trabalho objetiva analisar e descrever a distribuição dos casos notificados de sífilis em gestante no período de 2012 a 2017, no município de Codó.

\section{METODOLOGIA}

Trata-se de um estudo retrospectivo analítico, com base em levantamento de casos. O estudo compreende todos os casos notificados de sífilis em gestantes entre 2012 a 2017 no Município de Codó - Maranhão.

O município de Codó conta com uma população de 118.038 habitantes, sendo que 57.403 habitantes $48,65 \%$ são do sexo masculino e 60.635 habitantes - 51,35\% feminino, de acordo com o senso 2010 . $\mathrm{Na}$ última prévia realizada em 2017, a população foi e 120.810 habitantes. A cidade tem uma área territorial $1.026,384 \mathrm{~km}^{2}$, sua altitude em relação ao mar é de aproximadamente $47 \mathrm{~m}$, o clima predominante é o Tropical. Fuso horário UTC-3 (IBGE, 2008). Os dados demográficos gerais da área de abrangência foram obtidos do Instituto Brasileiro de Geografia e Estatística (IBGE), através de sua base de dados eletrônica (www.ibge.gov.br) e do Departamento de Informática do Sistema Único de Saúde (DATASUS).

Os dados foram coletados no Sistema de Informação de Agravos de Notificação - SINAN, contidos no Núcleo de Vigilância Epidemiológica (NVE) do município de Codó - Maranhão. Este estudo não envolveu diretamente pesquisa com seres humanos, pois foram utilizados dados secundários e públicos coletados para fins de O Sistema de Informação de Agravos de Notificação do Estado do Maranhão, sem a necessidade de apreciação ética por um Comitê de Ética especifico.

\section{RESULTADOS}

No período de 2012 a 2017 foram registrados 253 casos confirmados de sífilis em gestantes no município de Codó-MA, caracterizando uma média anual de 42,16 casos. O maior número de casos confirmados de sífilis em gestante foi no ano de 2016 ( $n=68 ; 26,88 \%)$ e 2015 ( $n=56 ; 22,14 \%)$. Já o ano de 2012 e 2013, foram registrados os menores números de casos confirmados, sendo eles $(n=20 ; 7,90 \%)$ e $(n=19 ; 7,90 \%)$, respectivamente. Os resultados podem ser observados na Tabela 1.

Tabela 1 - Casos confirmados de sífilis em gestantes no município de Codó no período de 2012 a 2017.

\begin{tabular}{ccc}
\hline Ano & N & $\%$ \\
\hline $\mathbf{2 0 1 2}$ & 20 & 7,90 \\
$\mathbf{2 0 1 3}$ & 19 & 7,50 \\
$\mathbf{2 0 1 4}$ & 43 & 17,00 \\
$\mathbf{2 0 1 5}$ & 56 & 22,14 \\
$\mathbf{2 0 1 6}$ & 68 & 26,88 \\
$\mathbf{2 0 1 7}$ & 47 & 18,58 \\
Total & 253 & 100 \\
\hline
\end{tabular}

Fonte: Ministério da Saúde/SVS - Sistema de Informação de Agravos e Notificação - Sinan Net, 2018. 
Os resultados da Tabela 2 mostram que a faixa etária com predominância dos casos confirmados de sífilis em gestante foram da faixa etária entre 20 e 39 anos ( $n=169 ; 66,80 \%$ ), enquanto que a idade com menor número de casos confirmados foi a compreendida entre 10 e 14 anos de idade ( $n=6 ; 2,37 \%)$.

Tabela 2 - Número de casos confirmados de sífilis em gestante no município de Codó segundo faixa etária, no período de janeiro de 2012 a dezembro de 2017.

\begin{tabular}{ccc}
\hline Faixa etária & $\mathbf{N}$ & $\%$ \\
\hline $\mathbf{1 0 - 1 4}$ & 6 & 2,37 \\
$\mathbf{1 5 - 1 9}$ & 68 & 26,88 \\
$\mathbf{2 0 - 3 9}$ & 169 & 66,80 \\
$\mathbf{4 0 - 5 9}$ & 10 & 3,95 \\
Total & 253 & 100 \\
\hline
\end{tabular}

Fonte: Ministério da Saúde/SVS - Sistema de Informação de Agravos e Notificação - Sinan Net, 2018.

Quanto a classificação clínica, a sífilis primária em gestantes foi a mais notificada ( $n=144 ; 56,92 \%)$. A segunda mais notificada foi a sífilis secundária $(n=26 ; 10,28 \%)$. Já o restante das classificações clínicas obteve percentual de casos confirmados próximo à zero, como pode ser observado na Tabela 3 . Vale ressaltar que o número de casos considerados como ignorados/ branco foi $78(30,83 \%)$.

Tabela 3 - Número de casos confirmados de sífilis em gestante no município de Codó segundo classificação clínica, no período de janeiro de 2012 a dezembro de 2017.

\begin{tabular}{lcc}
\hline Classificação clínica & $\mathbf{N}$ & $\%$ \\
\hline Ign/branco & 78 & 30,83 \\
Primária & 144 & 56,92 \\
Secundária & 26 & 10,28 \\
Terciária & 1 & 0,39 \\
Lactente & 4 & 1,58 \\
Total & 253 & 100 \\
\hline
\end{tabular}

Fonte: Ministério da Saúde/SVS - Sistema de Informação de Agravos e Notificação - Sinan Net, 2018.

Do total de 253 casos confirmados de sífilis em gestantes, 197 (77,86\%) foram considerados reativos segundo teste treponêmico e, apenas $4(1,58 \%)$ foram considerados como não reativo. Vale ressaltar que 0 número de casos ignorados/brancos e teste treponêmico não realizado correspondem a $20,56 \%$ dos casos, segundo a Tabela 4.

Tabela 4 - Número de casos confirmados de sífilis em gestante no município de Codó segundo Teste treponêmico, no período de janeiro de 2012 a dezembro de 2017.

\begin{tabular}{lcc}
\hline Teste treponêmico & $\mathbf{N}$ & $\%$ \\
\hline Ign/Branco & 18 & 7,12 \\
Reativo & 197 & 77,86 \\
Não reativo & 4 & 1,58 \\
Não realizado & 34 & 13,44 \\
Total & 253 & 100 \\
\hline
\end{tabular}

Fonte: Ministério da Saúde/SVS - Sistema de Informação de Agravos e Notificação - Sinan Net, 2018. 
O total de casos confirmados como reativo pelo teste não treponêmico foi 168 (66,40\%), enquanto os casos não reativos corresponderam a um percentual de 1,98\% $(n=5)$. Ainda segundo a Tabela $\mathbf{5}$, o número de casos ignorados/brancos e teste não realizado, corresponderam a um de percentual de $31,62 \%$ dos casos confirmados de sífilis em gestante no município de Codó.

Tabela 5 - Número de casos confirmados de sífilis em gestante no município de Codó segundo Teste nãotreponêmico, no período de janeiro de 2012 a dezembro de 2017.

\begin{tabular}{lcc}
\hline Teste não treponêmico & $\mathbf{N}$ & $\%$ \\
\hline Ign/Branco & 40 & 15,81 \\
Reativo & 168 & 66,40 \\
Não reativo & 5 & 1,98 \\
Não realizado & 40 & 15,81 \\
Total & 253 & 100 \\
\hline
\end{tabular}

Fonte: Ministério da Saúde/SVS - Sistema de Informação de Agravos e Notificação - Sinan Net, 2018.

\section{DISCUSSÃO}

Dada a importância dos casos de sífilis, este artigo pode ser usado como base para informação sobre inúmera variáveis relacionadas a doença, tais como: casos anuais, faixa etária das portadoras, classificação clínica e tipo de teste para diagnóstico entre 2012 e 2017 em Codó-MA. Trata-se de uma doença sexualmente transmissível, causada pelo Treponema pallidum, seja por relação sexual ou de mae para filho (transmissão vertical), sendo mais frequente em mulheres de idade fértil (CASAL; ARAÚJO; CORVELO, 2012; SUTO et al., 2016).

De acordo com a tabela 1, pode-se observar que no período observado foram 253 casos novos de sífilis, em um crescente, dados estes que estão de acordo aos de Moreira et al. (2017), que mostraram um aumento significativo nos últimos anos. A partir do biênio 2012-2013, percebeu-se que o número de casos teve aumento significativo, o que corrobora com o trabalho de Maciel et al (2017), que mostram que em uma cidade do interior de São Paulo houve um aumento do número de casos.

Em relação a idade das pacientes, de acordo com a tabela 2, a faixa etária de maior prevalência foi de 20 a 39, ratificando o que Silva et al (2017) encontraram em seus estudos, ambos os estudos justificam tal acontecimento pelo fato de as mulheres estarem no ápice da atividade sexual, bem como devido ao fato de estarem mais susceptíveis a sexo sem proteção, por conseguinte, elevam-se esses casos. Moreira et al (2017), foi mais sucinto e objetivo, e justificou tal achado em função do comportamento social das mulheres, portanto ações educativas que visem a diminuição do número de casos devem ser focadas em mulheres entre 20 e 39 anos.

Por estar em constante atividade sexual, esta torna-se a principal via de transmissão, já que a probabilidade da infecção fetal é influenciada pelo estágio da sífilis na mãe e a duração da exposição fetal , de acordo com Rocha et al. (2017), ou seja, a forma de transmissão é maior quando a mulher apresenta sífilis primária ou secundária durante a gestação, e como pode ser observado na tabela 3 , em relação à classificação clínica, a principal forma é a primária, que consiste no aparecimento de uma única lesão na área de entrada da bactéria, apresentando-se sem dor, rígida, com presença de secreção, vale ressalta que com o tempo a mesma regride de maneira espontânea (COELHO et al., 2018). Chama-se a atenção por cerca de $30,83 \%$ dos casos não terem classificação em relação a este aspecto, isso de acordo com Lima et al. (2017), se dá em função de casos subnotificados e que as equipes de atenção à saúde não conseguem acompanhar.

Para se propor estratégias para diminuir o número de casos de sífilis, e até mesmo propor estratégias de prevenção, necessita-se de métodos de diagnósticos rápidos e precisos, sejam eles treponêmicos (que buscam pelo agente etiológico) ou não. A fim de realizar um seguimento da sífilis na gestação é realizado um teste não treponêmico, geralmente o VDRL, e para fim de diagnóstico é necessário que sejam feitos pelo menos um de cada (COSTA et al., 2018). Como visto na tabela 4 , cerca de $77 \%$ dos casos foram reagentes para um teste treponemico, enquanto que cerca de $66 \%$ deram reagente para o teste não-treponemico, logo percebe-se que o tratamento foi capaz de reduzir uma parcela das gestantes com a doença, que se deu após o diagnóstico, esses dados corroboram com aqueles apresentados por Rocha et al. (2012). Uma sugestão para diagnóstico com maior velocidade e precisão foi proposto por Nascimento et al. (2018) que realizaram um estudo e mostraram que a implantação de testes rápidos para sífilis ainda está em andamento, e exige um grande esforço de vários departamentos do Ministério da Saúde e do município. 


\section{CONSIDERAÇÕES FINAIS}

Na cidade de Codó-MA, entre 2012 e 2017, foi observado uma crescente nos casos de sífilis em gestantes, com faixa etária entre 20 e 39, com manifestações primárias da doença e com realização de testes treponêmicos ou não, todos os dados desse estudo retrospectivo estão de acordo com aqueles previamente descritos na literatura, apesar de possíveis casos subnotificados, uma vez que se baseia em dados secundários, portanto são necessários novos estudos sobre essa temática em nossa região sejam realizados, com a finalidade de aprofundar os conhecimentos voltados para a necessidade de saúde dos pacientes com sífilis.

É nítido a necessidade da implementação de atividades educativas individuais e coletivas para acompanhamento das gestantes, buscando-se adesão ao diagnóstico, e ao uso regular do preservativo nas relações sexuais, haja vista tratar-se de uma doença curável, isso pode acontecer por meio da aproximação dos movimentos sociais, escolas e igrejas existentes na comunidade dentre outros, e teria como resultado a captação precoce de gestantes, para adoção de práticas de prevenção a doenças transmissíveis, para adesão das gestantes e parceiros aos tratamentos de doenças transmissíveis, e ao pré-natal.

Os registros de notificações de sífilis em gestantes são instrumentos valiosos para monitoramento e avaliação da assistência prestada às gestantes com diagnóstico de sífilis. Por isso, é importante que a gestão e a assistência entendam a necessidade do preenchimento adequado das informações do formulário e da alimentação do Sistema de Informação de Agravos de Notificação. Também é necessária a atualização dos profissionais para a assistência às gestantes com sífilis e a importância da notificação. O grande desafio para a saúde pública é aumentar a cobertura e a qualidade do pré-natal, incluindo o parceiro sexual ativamente nas consultas, ampliar o diagnóstico laboratorial da sífilis e o consequente tratamento durante o pré-natal e no momento do parto.

\section{REFERÊNCIAS}

1. CASAL CAD; ARAUJO, EC; CORVELO TCO. Aspectos imunopatogênicos da sífilis materno-fetal: revisão de literatura. Revista Paraense de Medicina, 2012; 26(2), 1-6.

2. CAVALCANTE PAM; PEREIRA RBL; CASTRO JGD. Sífilis gestacional e congênita em Palmas, Tocantins, 2007-2014. Epidemiologia e Serviços de Saúde, 2017; 26(2), 255-264.

3. COELHO JMR; DANTAS FCS; PENA LTG et al. Sífilis: um panorama epidemiológico do Brasil e do município de Volta Redonda/RJ. Brazilian Journal of Health Review, 2018 1(1), 128-147.

4. COSTA CC; FREITAS LV; SOUSA DMN. et al. Sífilis congênita no Ceará: análise epidemiológica de uma década. Revista da Escola de Enfermagem da USP, 2013; 47(1), 152-159.

5. FURTADO MFS; BRASIL GVS; ARAGÃO FBA et al. Fatores epidemiológicos da sífilis em gestantes no município de São Luís-MA. Revista Uningá, 2018, 52(1), 51-55.

6. GRUMACH AS; MATIDA LH; HEUKELBACH HLLC et al. A (des) Informação relativa à aplicação da penicilina na rede do sistema de saúde do Brasil: o caso da sífilis. Jornal Brasileiro de Doenças Sexualmente Transmissíveis, 2007; 19(3),120-127.

7. MACIEL, RB; BARROS IC; UGRINOVICH LA et al. Perfil epidemiológico dos casos de sífilis na cidade de Americana (SP) de 2005 a 2015. Revista de Epidemiologia e Controle de Infecção, 2017; 7(3), 161-168.

8. MOREIRA KFA; OLIVEIRA DM; ALENCAR LA et al. Perfil dos casos notificados de sífilis congênita. Cogitare e Enfermagem, 2017; 22(2), 1-10.

9. NASCIMENTO DSF; SILVA RC; TÁRTARI DO et al. Relato da dificuldade na implementação de teste rápido para detecção de sífilis em gestantes na Atenção Básica do SUS em um município do Sul do Brasil. Revista Brasileira de Medicina de Família e Comunidade, 2018; 13(40), 1-8.

10. REZENDE, EMA; BARBOSA, NB. A sífilis congênita como indicador da assistência de pré-natal no Estado de Goiás. Revista de APS, 2015; 18(2), 220-232.

11. ROCHA RPS; TERÇAS ACP; NASCIMENTO VF et al. Análise do perfil epidemiológico de sífilis nas gestantes em Tangará Da Serra de 2007 a 2014. Renome, 2017; 5(2), 3-21.

12. SILVA, LCVG; TEODORO CCJ; SILVA JK et al. Perfil dos casos de sífilis congênita em um município do sul de Mato Grosso. Journal Health NPEPS, 2017; 2(2), 380-390.

13. SUTO CSS; SILVA DL; ALMEIDA LS et al. Assistência pré-natal a gestante com diagnóstico de sífilis. Revista de Enfermagem e Atenção à Saúde, 2016; 5(2), 18-33.

14. VASCONCELOS MIO; OLIVEIRA KMC; MAGALHÃES AHR et al. Sífilis na gestação: estratégias e desafios dos enfermeiros da atenção básica para o tratamento simultâneo do casal. Revista Brasileira em Promoção da Saúde, 2017; 29(sup.), 85-92. 